\title{
Bilateral brown tumors of maxilla with primary hyperparathyroidism
}

Volume 8 Issue 4 - 2017

Keywords: hyperparathyroidism, granuloma, brown tumors, osteoporosis

\section{Introduction}

In the past four decades, the practice of checking serum calcium on routine blood screens has led to earlier diagnosis of primary hyperparathyroidism. It has become increasingly rare to find advanced disease like generalized osteoporosis, multiple focal areas of demineralization of the skull or osteitis fibrosa cystica with brown tumour, as the manifestations of this disease. It is especially rare for advanced bone disease, such as brown tumour, to present in the absence of other symptoms of hypercalcaemia in Indian population.

\section{Case report}

A 46-year-old woman presented with a 7years history of an enlarging mass beneath the right cheek. She had a history of a similar mass beneath the left cheek 5years ago, which spontaneously decreased in size. She had a history of gradually increasing bilateral nasal obstruction since 6years. The patient denied previous trauma, epistaxis or ocular changes. A complete review of systems was unremarkable, with absence of any symptoms of hypercalcaemia such as fatigue, malaise, joint pain, polyuria, or abdominal discomfort. She did not give any history suggestive of renal failure or bone. She gave history of previous surgery sublabially biopsy of which showed a giant cell reparative granuloma.

On examination, a $7-8 \mathrm{~cm}$ sized non-tender, immobile, smooth surfaced, hard mass was palpated beneath the right nasolabial fold. Another mass around $1-1.5 \mathrm{~cm}$ in size was palpated beneath the left nasolabial fold (Figure 1). Anterior rhinoscopy revealed a round, mucosal-covered mass extending along the floor and lateral wall of nose into the right nasal vestibule. No proptosis or extra-ocular muscle involvement was noted. Examination of the oral cavity was unremarkable except for a bulge in the upper gingivolabial sulcus on right side. No Cervical adenopathy was apparent.

Acomputed tomography scan of the paranasal sinuses demonstrated an expansile multiloculated osteolytic lesion with soft tissue density within, involving both the maxillae (Figure 2). With the diagnosis of a metabolic disease, laboratory investigations revealed a high serum calcium $(12.9 \mathrm{mg} / \mathrm{dl})$ and a low phosphate level $(2.1 \mathrm{mg} / \mathrm{dl})$, a raised 24 hour urine calcium of $411 \mathrm{mg} /$ day and a markedly raised urine calcium/creatinine ratio of 0.65 (normal $<0.2$ ) and a normal $25(\mathrm{OH})$ ) VIT D levels.

The serum parathyroid hormone levels were elevated above 1900 $\mathrm{pg} / \mathrm{ml}$. The other blood investigations like urea nitrogen, creatinine, glucose, bilirubin, uric acid, protein, albumin, globulin, serum aspartate aminotransferase, and thyroid-stimulating hormone levels were all normal. The serum immunoelectrophoresis was normal. The concentrations of $\operatorname{IgG}, \operatorname{IgA}$, and $\operatorname{IgM}$ were normal. With a working diagnosis of primary hyperparathyroidism with maxillary brown

\section{Kiruba Shankar Manoharan,' Bhuvaneshwari Venkatesan, ${ }^{2}$ Sakthi Saranya, ${ }^{3}$ Shanmugasundaram $\mathrm{PN}^{4}$}

'Department of Otolaryngology Employee state insurance medical college and post graduate institute of medical studies and research, India

${ }^{2}$ Department of Radiology Employee state insurance medical college and post graduate institute of medical studies and research, India

${ }^{3}$ Department of Dermatology Madras Medical College, India ${ }^{4}$ Department of General surgery Kilpauk Medical College, India

\begin{abstract}
Correspondence: Kiruba Shankar Manoharan Department of Otolaryngology Employee state insurance medical college and post graduate institute of medical studies and research Chennai, India,Tel 08973618016,Email drkirubashankar@gmail.com
\end{abstract}

Received: November 14, 2016 | Published: September 13, 2017

tumours, a 99mTC-MIBI parathyroid scan was done localized a lesion to the left inferior parathyroid gland (Figure 3).

A DEXA scan showed low Z scores and generalized osteoporosis. A 99mTC-MDP bone scan showed brown tumors involving bilateral maxilla and left iliac bone. The patient was maintained on salt rich diet and adequate fluid intake until which the patient was planned for parathyroid exploration. A sublabial biopsy from the maxillary lesion showed a giant cell lesion probably a reparative granuloma.

A neck exploration was performed and a $2 \times 1.8 \mathrm{~cm}$ lesion was found involving the left inferior parathyroid gland. The lesion was excised and histo-pathological examination revealed a parathyroid adenoma. The patient developed post-operative hypocalcaemia suggestive of a hungry bone syndrome. The patient was managed with calcium supplements. The patient recovered well. A 6month follow-up of the patient showed a remarkable regression in the maxillary brown tumors.

\section{Discussion}

Various anatomical and pathological entities, both benign and malignant, can appear as bone-expanding or lytic lesions in the facial bones. ${ }^{1}$ In the case of a lytic region of the jaw bones, the most likely diagnoses would include: odontogenic cysts and tumors (radicular cyst, lateral periodontal cyst, and ameloblastoma), infectious diseases (bone abscess, localized osteomyelitis), metabolic bone disease hyperparathyroidism, metastasis from a known or an unknown primary site (lung, breast, kidney, prostate), primary bone tumors and cysts (simple bone cyst, eosinophilic granuloma, giant cell lesions, odontogenic keratocyst, myxoma and odontogenic fibroma). ${ }^{2}$ 


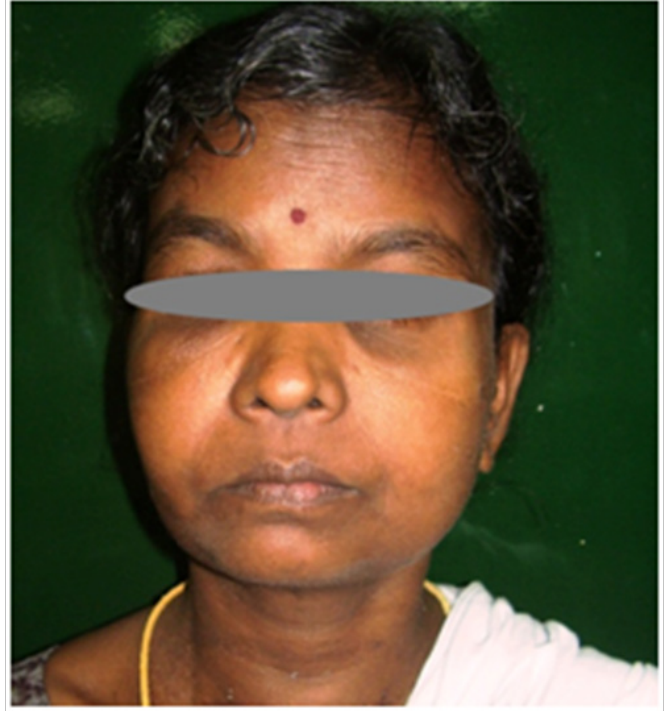

Figure I Clinical picture demonstrating the subtle fullness in the cheek.

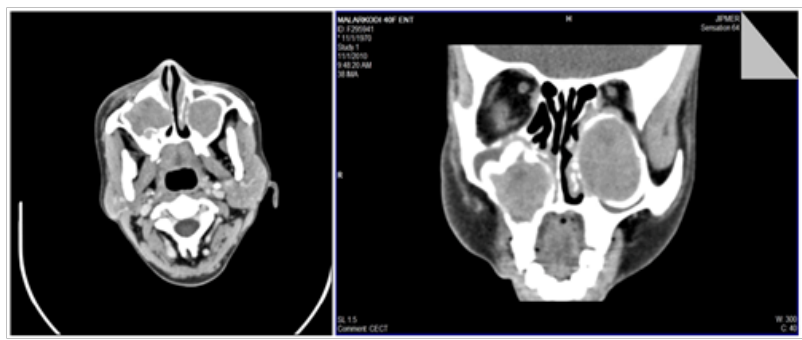

Figure 2 Axial and coronal section of computed tomography of bilatera maxillary tumors. It demonstrates expansile multiloculated osteolytic lesion with soft tissue density within, involving both the maxillae.

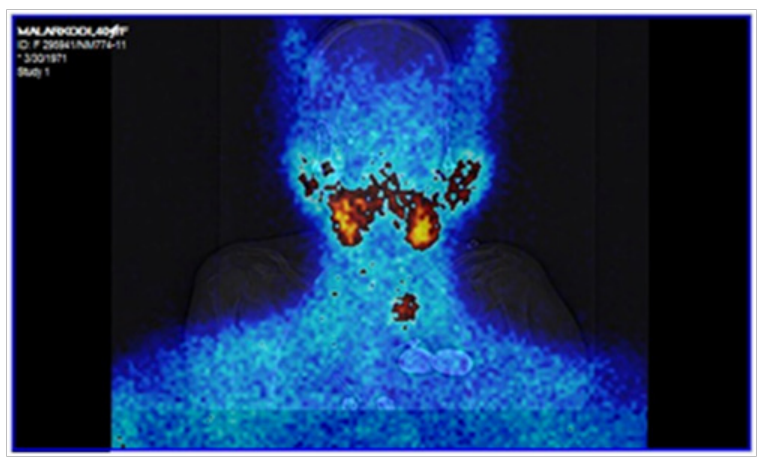

Figure 3 Tc-MIBI radionucleotide uptake study revealed an active parathyroid adenoma. Normal uptake in the submandibular gland is also noted.

Bone-expanding giant cell lesions that can arise in the jaw bones include giant cell tumor, giant cell reparative granuloma, cherubism and brown tumor. As it is difficult to distinguish brown tumors from other giant cell lesions on the basis of histology or radiology examination, the clinical diagnosis is made based in relation to hyperparathyroidism.

The brown tumor of hyperparathyroidism is a localized bone tumor and an uncommon manifestation of primary Hyperparathyroidism. The role of PTH is to control calcium and phosphorous levels in the plasma and in the extracellular fluid and this is done partly by modulating the balance between osteoblastic and osteoclastic activities.
In hyperparathyroid states, an imbalance exists between osteoblastic and osteoclastic activity. Lamellar bone is being constantly resorbed while woven bone is laid down to replace it. Occasionally soft tissue proliferation replaces the lost bone. As a result of the direct effect of PTH on bones, a conversion of the osteogenic potential of the cells occurs changing them from osteoblasts to osteoclasts, with bone resorption predominating over the formation of new bone tissue. As a result of intraosseous bleeding and tissue degeneration, cysts may develop; groups of hemosiderin loaded macrophages, giant cells and fibroblast fill these cystic lesions. The hemorrhages and hemosiderin deposits in the tumors give rise to the characteristic color of the lesions and the term brown tumors.

Over the past two decades brown tumour has been a rare manifestation of primary hyperparathyroidism. The reported prevalence of brown tumors is $0.1 \%{ }^{3}$ Brown tumors arise secondary to both primary and secondary hyperparathyroidism, and have been reported to occur in $4.5 \%$ of patients with primary hyperparathyroidism and in $1.5-1.7 \%$ of those with secondary disease. ${ }^{4}$ The growing use of biochemical assays has led to an earlier diagnosis of the disease as asymptomatic hypercalcaemia.

However, brown tumour can be the first manifestation of hyperparathyroidism, usually incurred in the ribs, clavicle and pelvic girdle. ${ }^{5}$ Less commonly there is facial bone involvement, and mandibular and maxillary bones are the facial bones that are usually involved. ${ }^{6}$ When a tumor of the maxilla has been diagnosed as a giant cell tumor, hyperparathyroidism should be ruled out to exclude the possibility that it is a brown tumor. ${ }^{7}$

The initial treatment of brown tumor involves control of hyperparathyroidism, regardless of whether it is primary or secondary. Treatment of primary hyperparathyroidism requires a parathyroidectomy. Once hyperparathyroidism is controlled, the tumor tends to regress, although in some cases surgical removal is necessary, especially for patients who have large, symptomatic tumors. Brown tumor can recur if hyperparathyroidism persists or recurs. Noleto et al., ${ }^{5}$ demonstrated that bone lesions regress spontaneously following removal of the diseased parathyroid gland; however Yamazaki et al. recommend curettage and enucleation. There was a spontaneous regression of the tumor in the present case.

\section{Conclusion}

Brown tumors of the jaw rarely involve the maxilla in association with primary hyperparathyroidism. The presence of underlying primary hyperparathyroidism should be sought in all unexplained bilateral maxillary swellings. The patients do quite well with parathyroid adenoma excision. However giant residual on nonregressing tumors may need surgical excision or curettage.

\section{Financial disclosure}

There are no financial disclosures to be made regarding this report.

\section{Conflicts of interest}

The authors have no conflict of interest or financial relationship to reveal.

\section{Acknowledgments}

None. 


\section{Funding}

None.

\section{References}

1. Mirra JM. Bone tumor. Clinical, Radiologic, and Pathologic Correlations. Lea \& Febiger, Philadelphia, PA, USA. 1989. p.1785.

2. Proimos E, Chimona TS, Tamiolakis D, et al. Brown tumor of the maxillary sinus in a patient with primary hyperparathyroidism: a case report. J Med Case Rep. 2009;3:7495.

3. Rai S, Rattan V, Bhadada SK. Giant Cell Lesions Associated with Primary Hyperparathyroidism. J Maxillofac Oral Surg. 2015;14(4):930-2934.

4. Nunes TB, Bologna SB, Witzel AL, et al. Rare Case of Concomitant Maxilla and Mandible Brown Tumours, Papillary Thyroid Carcinoma, Parathyroid Adenoma, and Osteitis Fibrosa Cystica. Case Reports in Dentistry. 2016;2016(2016):4.
5. Noleto JW, Ramos IA, Rocha JF, et al. A rare case of regression of brown tumors of tertiary hyperparathyroidism after parathyroidectomy and renal transplant: A 5-year follow-up. Ann Maxillofac Surg. 2016;6(1):125-159.

6. Queiroz IV, Queiroz SP, Medeiros RJ,et al. Brown tumor of secondary hyperparathyroidism: surgical approach and clinical outcome. Oral Maxillofac Surg. 2016;20(4):435-439.

7. Jebasingh F, Jacob JJ, Shah A, et al. Bilateral maxillary brown tumors as the first presentation of primary hyperparathyroidism. Oral Maxillofac Surg. 2008;12(2):97-100. 